\title{
Isochronal function projective synchronization between chaotic and time-delayed chaotic systems
}

\author{
Ranchao $\mathrm{Wu}^{*}$ and Junlian Liu
}

* Correspondence: rcwu@ahu.edu. cn

School of Mathematics, Anhui University, Hefei 230039, P. R. China

\begin{abstract}
Isochronal function projective synchronization between chaotic and time-delayed chaotic systems with unknown parameters is investigated in this article. Based on Lyapunov stability theory, adaptive controllers and parameter updating laws are designed to achieve the isochronal function projective synchronization between chaotic and time-delayed chaotic systems. The scheme is applied to realize the synchronization between time-delayed Lorenz systems and time-delayed hyperchaotic Chen systems, respectively. Numerical simulations are also presented to show the effectiveness of the proposed method.

Mathematics Subject Classification 2000: 34C28; 34D20; 37N35.
\end{abstract}

Keywords: isochronal function projective synchronization, time-delayed chaotic systems, adaptive control

\section{Introduction}

In the last few years, chaos synchronization has gained a lot of attention for its potential applications in some engineering applications, such as image processing, chemical and biological systems, information science and in particular secure communication. Since the pioneering work of Pecora and Carroll [1], in which complete synchronization between two identical chaotic systems with different initial conditions was realized, various approaches have been put forward for synchronization of chaotic systems, such as complete synchronization [2], phase synchronization [3], generalized synchronization [4], lag synchronization [5], projective synchronization [6], modified projective synchronization $[7,8]$ and function projective synchronization [9-11], function projective lag synchronization [12], anti-synchronization [13] and so on.

Among all types of chaos synchronization, projective synchronization phenomenon is of great significance for its potential application in secure communication. In 1999, Mainieri and Rehacek [14] first proposed the concept of projective synchronization, which is characterized that the drive and the response systems could be synchronized up to a scaling factor. Because of the proportionality between its synchronized dynamical states, the feature can be used to $M$-nary digital communication for achieving fast communication. So projective synchronization have attracted increasing attention during recent years and some conditions ensuring projective synchronization have been obtained. Recently, some scholars extended the concept of projective synchronization and proposed modified projective synchronization [15], function projective

(c) 2012 Wu and Liu; licensee Springer. This is an Open Access article distributed under the terms of the Creative Commons Attribution License (http://creativecommons.org/licenses/by/2.0), which permits unrestricted use, distribution, and reproduction in any medium, provided the original work is properly cited. 
synchronization [16] and modified function projective synchronization $[17,18]$, in which master and slave system are synchronized with a scaling function matrix.

As we know, delayed differential equations could exhibit complex dynamical behaviors and have attracted much attention in the field of nonlinear dynamics. Note that research on synchronization between time-delayed chaotic systems has been extensively carried out, see, for example, [19-21]. Recently, dual-anticipating, dual and duallag synchronization [22] between two identical time-delayed chaotic systems, lag synchronization [23] were investigated, where lag or anticipatory dynamics occurred, i.e., there existed a lag time or anticipatory time phase shift between state vectors. While results about zero lag time difference between synchronized state shifts, i.e., isochronal synchronization $[24,25]$ are also obtained between time-delayed systems. Note that projective synchronization [26,27] between time-delayed chaotic systems was extensively investigated. However, results about isochronal function projective synchronization between chaotic and time-delayed chaotic systems are still few. In this article, isochronal function projective synchronization scheme between chaotic and timedelayed chaotic systems with unknown parameters is proposed. The method is shown to be effective by applying to Lorenz and hyper-chaotic Chen systems.

The remainder of this article is organized as follows. In Section 2 the synchronization scheme is presented. Section 3 is devoted to the application of the proposed scheme to Lorenz and hyper-Chaotic systems, respectively. Numerical simulations are also presented to demonstrate the effectiveness of the method. Some conclusions are drawn in Section 4.

\section{Statement of the problem}

Consider the chaotic system given by

$$
\dot{x}=F(x) \theta+G(x) \beta+f(x),
$$

where $x \in R^{n}$ denotes the state vector, $F, G: R^{n} \rightarrow R^{n \times p}$ are continuous function matrices, $f: R^{n} \rightarrow R^{n}$ is a continuous nonlinear vector function, $\theta, \beta \in R^{p}$ are parameter vectors. Note that many chaotic and hyper-chaotic systems, such as Lorenz system, Chen system, Lü system, Rössler system, hyper-chaotic Chen system, etc, could be described by system (1).

The time-delayed version of system (1) could also exhibit chaotic behaviors, such as time-delayed Lorenz system [19] and time-delayed Rossler system [21], etc. To consider the isochronal synchronization between chaotic and time-delayed chaotic systems, take the drive system as follows

$$
\dot{y}=F(y) \theta_{r}+G(y-\tau) \beta_{r}+f(y) .
$$

The response system is

$$
\dot{x}=F(x) \theta+G(x) \beta+f(x)+u,
$$

where $u=\left(u_{1}, u_{2}, \ldots, u_{n}\right)^{T} \in R^{n}$ is the control input to be determined later.

Now we need to design the controller $u$ such that the chaotic system (1) could track the trajectory of time-delayed system (2). Define the error $e=x-\lambda(t) y$, where $\lambda(t)=$ $\operatorname{diag}\left(\lambda_{1}(t), \lambda_{2}(t), \ldots, \lambda_{n}(t)\right)$.

Definition 1. It is said that isochronal function projective synchronization occurs between systems (1) and (2) if there exists a diagonal function matrix $\lambda(t)$ such that 


$$
\lim _{t \rightarrow \infty}\|e\|=\lim _{t \rightarrow \infty}\|x-\lambda(t) y\|=0 .
$$

To achieve the isochronal synchronization between (1) and (2) with different initial conditions, now the control input is chosen as

$$
\begin{aligned}
u= & -F(x) \theta-G(x) \beta-f(x)+\lambda(t)(F(y) \theta+G(y-\tau) \beta+f(y)) \\
& +\dot{\lambda}(t) y-k e,
\end{aligned}
$$

where $k=\operatorname{diag}\left(k_{1}, k_{2}, \ldots, k_{n}\right), k_{i}>0(i=1,2, \ldots, n)$ are constants. Consequently, we get

$$
\dot{e}=\lambda(t) F(\gamma)\left(\theta-\theta_{r}\right)+\lambda(t) G(\gamma-\tau)\left(\beta-\beta_{r}\right)-k e .
$$

The parameter updating laws for $\theta_{r}, \beta_{r}$ are chosen as

$$
\left\{\begin{array}{l}
\dot{\theta}_{r}=\lambda(t) e F(y) \\
\dot{\beta}_{r}=\lambda(t) e G(y-\tau)
\end{array}\right.
$$

then the synchronization result follows immediately.

Theorem 1. Isochronal function projective synchronization between systems (1) and (2) will occur under the control (4) and parameter updating laws (6).

Proof. Take the Lyapunov function

$$
V=\frac{1}{2}\left(e^{T} e+e_{\theta}^{T} e_{\theta}+e_{\beta}^{T} e_{\beta}\right),
$$

where $e_{\theta}=\theta-\theta_{r}, e_{\beta}=\beta-\beta_{r}$. The time derivative of $V$ along the trajectory of error system (5) is

$$
\begin{aligned}
\dot{V} & =e^{T} \dot{e}+e_{\theta}^{T} \dot{e}_{\theta}+e_{\beta}^{T} \dot{e}_{\beta} \\
& =e^{T} \dot{e}+e_{\theta}^{T}\left(-\dot{\theta}_{r}\right)+e_{\beta}^{T}\left(-\dot{\beta}_{r}\right) .
\end{aligned}
$$

In view of systems (5) and (6), one has

$$
\dot{V}=-\sum_{i=1}^{n} k_{i} e_{i}^{2} \leq 0 .
$$

So by Lyapunov stability theory, $e_{i} \rightarrow 0$ as $t \rightarrow \infty$, i.e., the synchronization will occur.

Remark 1. When $\lambda(t)=I$, $-I$, the complete synchronization and anti-synchronization between (1) and (2) are achieved, respectively. When $\lambda(t)=\alpha I$, $\operatorname{diag}\left(\lambda_{1}, \ldots, \lambda_{n}\right)$, then generalized projective synchronization and modified projective synchronization between (1) and (2) will happen, respectively.

Remark 2. Here structures of systems (1) and (2) are in the same form. Similarly, the synchronization result could also hold between chaotic and delayed chaotic systems with different structures under appropriate controllers and parameter updating laws.

\section{Applications}

\subsection{FPS between Lorenz and delayed Lorenz systems}

The delayed Lorenz system is taken as the drive system

$$
\left\{\begin{array}{l}
\dot{y}_{1}=a_{r}\left(y_{2}-y_{1}\right) \\
\dot{y}_{2}=b_{r} y_{1}-y_{2}-y_{2} y_{3} \\
\dot{y}_{3}=y_{1} y_{2}-c_{r} y_{3}(t-\tau)
\end{array}\right.
$$

where $a_{r}, b_{r}, c_{r}$ are uncertain parameters to be estimated. The system exhibits chaotic behaviors when $a_{r}=10, b_{r}=28, c_{r}=\frac{8}{3}$ and $\tau=0.3$, see Figure 1 . 


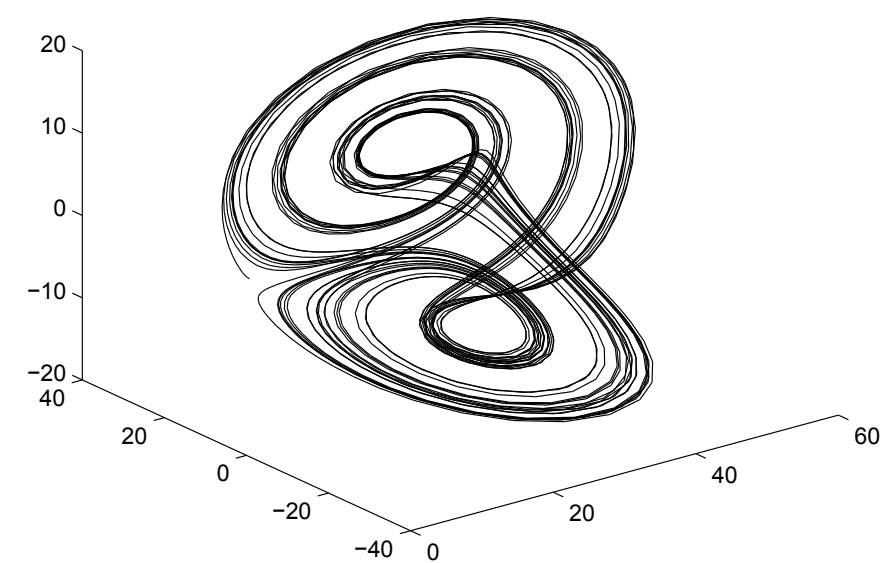

Figure 1 Chaotic attractor of time-delayed Lorenz system.

The response system is the Lorenz system with controllers

$$
\left\{\begin{array}{l}
\dot{x}_{1}=a\left(x_{2}-x_{1}\right)+u_{1} \\
\dot{x}_{2}=b x_{1}-x_{2}-x_{2} x_{3}+u_{2} \\
\dot{x}_{3}=x_{1} x_{2}-c x_{3}+u_{3} .
\end{array}\right.
$$

Define the errors $e_{i}=x_{i}-\lambda_{i}(t) y_{i}, i=1,2,3$. Choose the controllers as follows

$$
\left\{\begin{array}{l}
u_{1}=-a\left(x_{2}-x_{1}\right)+\lambda_{1}(t) a\left(y_{2}-y_{1}\right)+\dot{\lambda}_{1}(t) y_{1}-k_{1} e_{1}, \\
u_{2}=-b x_{1}+x_{2}+x_{2} x_{3}+\lambda_{2}(t)\left(b y_{1}-y_{2}-y_{1} y_{3}\right)+\dot{\lambda}_{2}(t) y_{2}-k_{2} e_{2}, \\
u_{3}=-x_{1} x_{2}+c x_{3}+\lambda_{3}(t)\left(y_{1} y_{2}-c y_{3}(t-\tau)\right)+\dot{\lambda}_{3}(t) y_{3}-k_{3} e_{3},
\end{array}\right.
$$

where $k_{i}>0, i=1,2,3$, then from systems (9), (10), and (11) one has

$$
\left\{\begin{array}{l}
\dot{e}_{1}=\lambda_{1}(t)\left(a-a_{r}\right)\left(y_{2}-y_{1}\right)-k_{1} e_{1} \\
\dot{e}_{2}=\lambda_{2}(t)\left(b-b_{r}\right) y_{1}-k_{2} e_{2} \\
\dot{e}_{3}=-\lambda_{3}(t)\left(c-c_{r}\right) y_{3}(t-\tau)-k_{3} e_{3} .
\end{array}\right.
$$

The parameter updating laws are given by

$$
\left\{\begin{array}{l}
\dot{a}_{r}=\lambda_{1}(t)\left(y_{2}-y_{1}\right) e_{1} \\
\dot{b}_{r}=\lambda_{2}(t) y_{1} e_{2} \\
\dot{c}_{r}=-\lambda_{3}(t) y_{3}(t-\tau) e_{3} .
\end{array}\right.
$$

Along the way similar to that of Theorem 1, one could arrive the following result.

Theorem 2. Isochronal function projective synchronization between Lorenz system (10) and delayed Lorenz system (9) will be realized under the controllers (11) and parameter updating laws (13).

\subsection{FPS between hyper-chaotic Chen and delayed hyper-chaotic Chen systems}

Consider the delayed hyper-chaotic Chen system

$$
\left\{\begin{array}{l}
\dot{y}_{1}=a_{r}\left(y_{2}-y_{1}\right)+y_{4} \\
\dot{y}_{2}=d_{r} y_{1}-y_{1} y_{3}+c_{r} y_{2}(t-\tau) \\
\dot{y}_{3}=b_{r} y_{3}-y_{1} y_{2} \\
\dot{y}_{4}=p_{4} y_{4}+y_{2} \gamma_{3}
\end{array}\right.
$$

When $a_{r}=35, b_{r}=3, c_{r}=12, d_{r}=7, p_{r}=0.5$, and $\tau=0.4$, then the system displays chaotic behaviors, see Figure 2 . 
The response system is the hyper-chaotic Chen system with controllers

$$
\left\{\begin{array}{l}
\dot{x}_{1}=a\left(x_{2}-x_{1}\right)+x_{4}+u_{1} \\
\dot{x}_{2}=d x_{1}-x_{1} x_{3}+c x_{2}+u_{2} \\
\dot{x}_{3}=b x_{3}-x_{1} x_{2}+u_{3} \\
\dot{x}_{4}=p x_{4}+x_{2} x_{3}+u_{4}
\end{array}\right.
$$

Define the errors $e_{i}=x_{i}-\lambda_{i}(t) y_{i}, i=1,2,3,4$. Choose the controllers as follows

$$
\left\{\begin{aligned}
u_{1}= & -a\left(x_{2}-x_{1}\right)-x_{4}+\lambda_{1}(t)\left(a\left(y_{2}-y_{1}\right)+y_{4}\right)+\dot{\lambda}_{1}(t) y_{1}-k_{1} e_{1}, \\
u_{2}= & -d x_{1}+x_{1} x_{3}-c x_{2}+\lambda_{2}(t)\left(d y_{1}-y_{1} y_{3}+c_{2} y_{2}(t-\tau)\right) \\
& +\dot{\lambda}_{2}(t) y_{2}-k_{2} e_{2} \\
u_{3}= & -x_{1} x_{2}+b x_{3}+\lambda_{3}(t)\left(y_{1} y_{2}-b y_{3}\right)+\dot{\lambda}_{3}(t) y_{3}-k_{3} e_{3} \\
u_{4}= & -p_{4} y_{4}-x_{2} x_{3}+\dot{\lambda}_{4}(t) y_{4}+\lambda_{4}(t)\left(p y_{4}+y_{2} y_{3}\right)-k_{4} e_{4}
\end{aligned}\right.
$$

where $k_{i}>0, i=1,2,3,4$, then from systems (14), (15), and (16) one has

$$
\left\{\begin{array}{l}
\dot{e}_{1}=\lambda_{1}(t)\left(a-a_{r}\right)\left(y_{2}-y_{1}\right)-k_{1} e_{1} \\
\dot{e}_{2}=\lambda_{2}(t)\left(c-c_{r}\right) y_{2}(t-\tau)+\lambda_{2}(t)\left(d-d_{r}\right) y_{1}-k_{2} e_{2} \\
\dot{e}_{3}=-\lambda_{3}(t)\left(b-b_{r}\right) y_{3}-k_{3} e_{3} \\
\dot{e}_{4}=\lambda_{4}(t)\left(p-p_{4}\right) y_{4}-k_{4} e_{4}
\end{array}\right.
$$

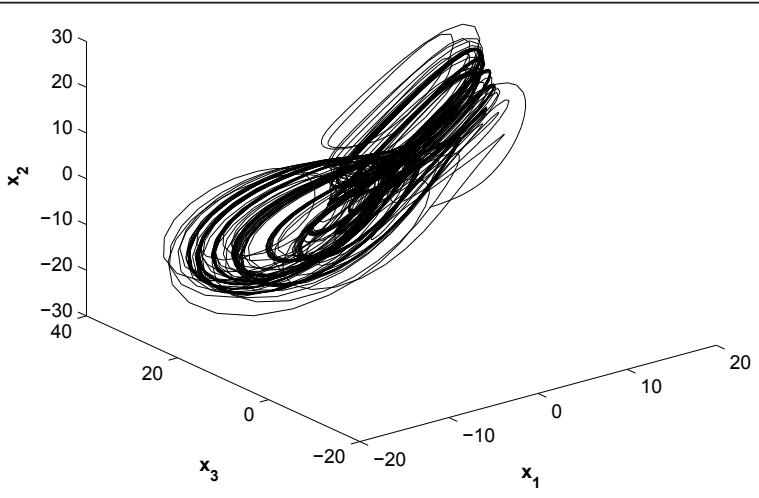

(a)

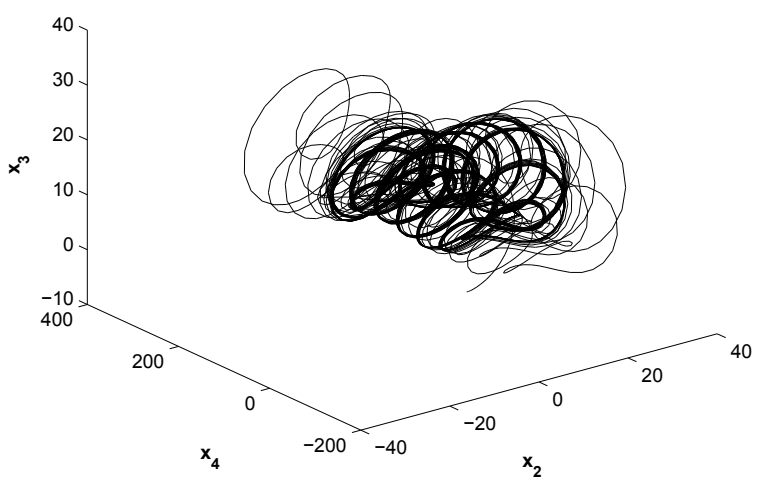

(b) 
The parameter updating laws are given by

$$
\left\{\begin{array}{l}
\dot{a}_{r}=\lambda_{1}(t)\left(y_{2}-y_{1}\right) e_{1} \\
\dot{b}_{r}=-\lambda_{3}(t) y_{3} e_{3} \\
\dot{c}_{r}=\lambda_{2}(t) y_{2}(t-\tau) e_{2} \\
\dot{d}_{r}=\lambda_{2}(t) y_{1} e_{2} \\
\dot{p}_{r}=\lambda_{4}(t) y_{4} e_{4} .
\end{array}\right.
$$

As a result, the synchronization between (14) and (15) will happen.

Theorem 3. Isochronal function projective synchronization between hyper-chaotic Chen system (15) and delayed hyper-chaotic Chen system (14) will be achieved under the controllers (16) and parameter updating laws (18).

The proof is similar to that of Theorem 1.

\subsection{Numerical simulations}

The initial conditions for systems (9) and (10) are respectively chosen as $\left(y_{1}(0), y_{2}(0)\right.$, $\left.y_{3}(0)\right)=(1,-3,2)$ and $\left(x_{1}(0), x_{2}(0), x_{3}(0)\right)=(-1,3,-2)$, and let $\tau=0.3$. The scaling functions are $\lambda_{1}(t)=\sin (t), \lambda_{2}(t)=\cos (t), \lambda_{3}(t)=-\sin (t)$. Moreover, $\left(k_{1}, k_{2}, k_{3}\right)=(1,3,2)$. The simulation results are shown in Figure 3 . Note that the error variables $e_{1}, e_{2}, e_{3}$ tend to zero and the estimated values of unknown parameters $a_{r}, b_{r}, c_{r}$ converge to $10,28, \frac{8}{3}$, respectively.

The initial conditions for systems (14) and (15) are respectively chosen as $\left(y_{1}(0), y_{2}\right.$ $\left.(0), y_{3}(0), y_{4}(0)\right)=(-3,4,-2,-2)$ and $\left(x_{1}(0), x_{2}(0), x_{3}(0), x_{4}(0)\right)=(3,-4,2,2)$, and let $\tau=0.3$. The scaling functions are $\lambda_{1}(t)=\sin (t), \lambda_{2}(t)=-\cos (t), \lambda_{3}(t)=\cos (t), \lambda_{4}(t)=-\sin (t)$. Moreover, the control gains are chosen as $\left(k_{1}, k_{2}, k_{3}, k_{4}\right)=(7,5,5,5)$. The simulation results are shown in Figures 4 and 5 . Note that the error variables tend to zero and
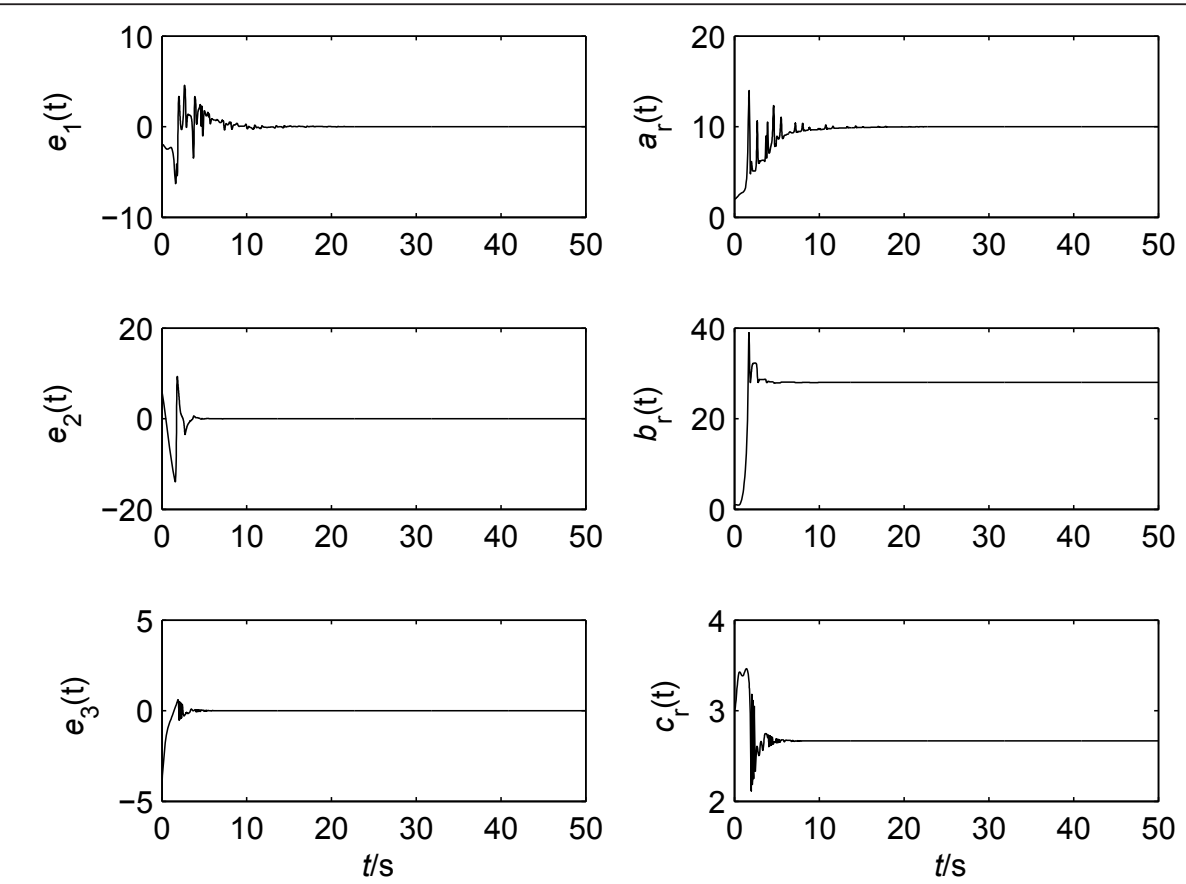

Figure 3 Time evolution of errors and estimated values of unknown parameters. 

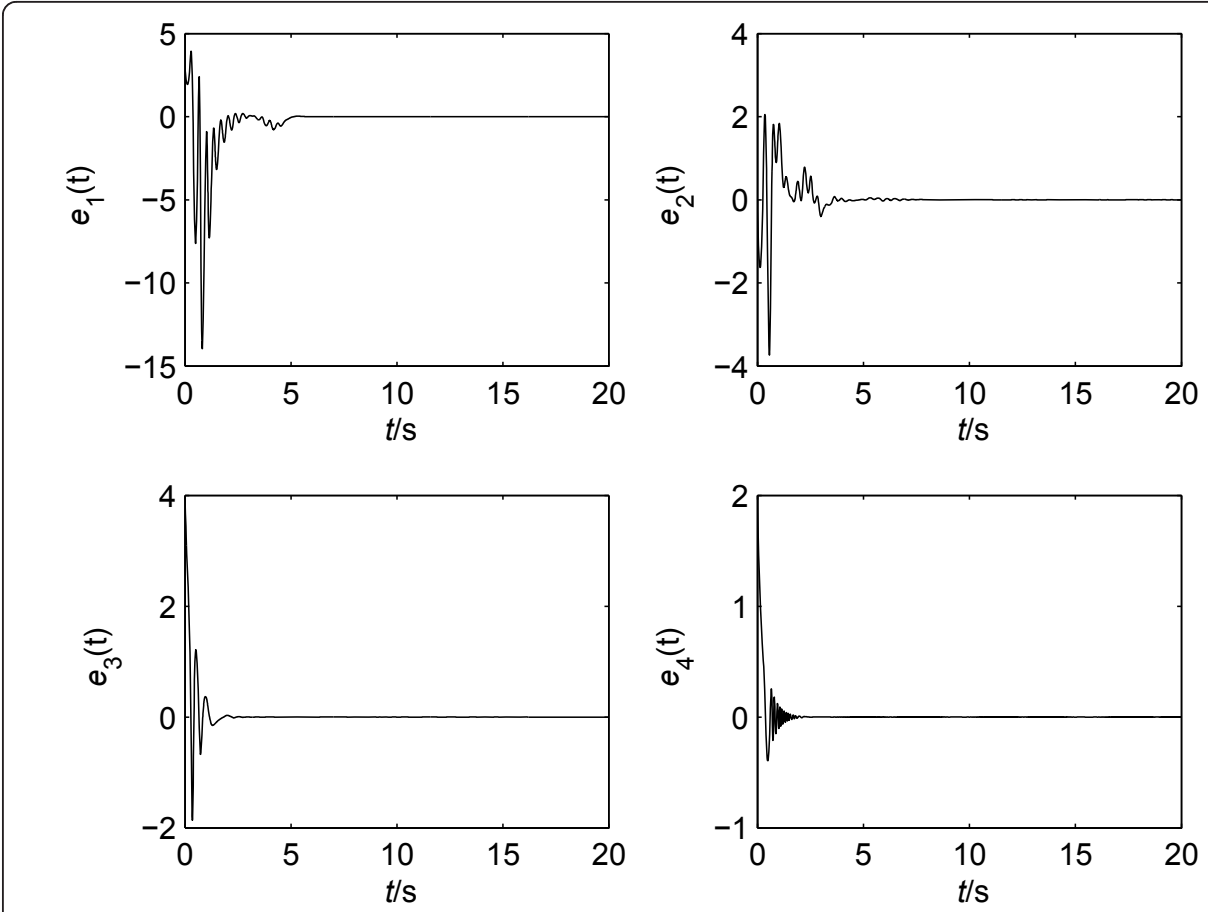

Figure 4 Time evolution of error variables of systems (18).
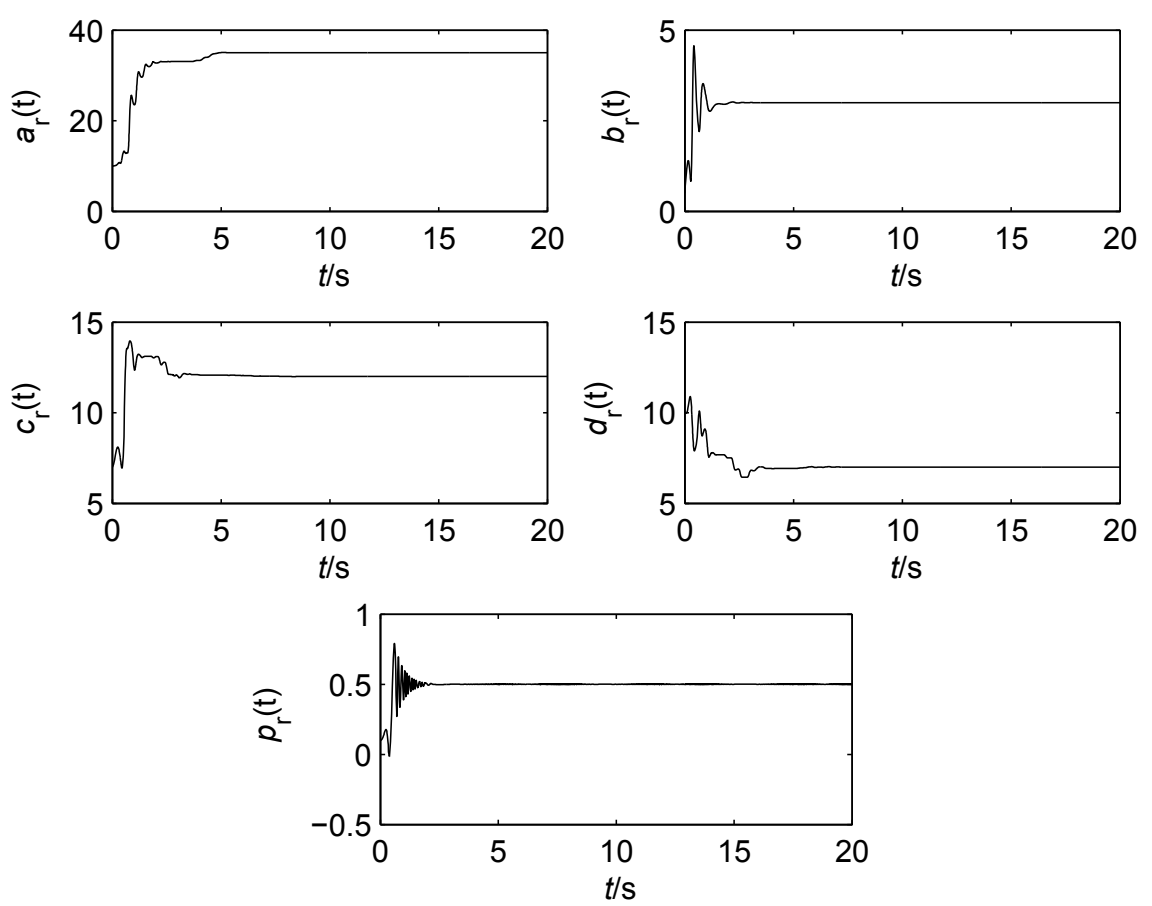

Figure 5 Time evolution of unknown parameters of system (19). 
the estimated values of unknown parameters $a_{r}, b_{r}, c_{r}, d_{r}, p_{r}$ converge to $35,3,12,7$, 0.5 , respectively.

\section{Conclusions}

In this article, function projective synchronization between chaotic and time-delayed chaotic systems with unknown parameters is investigated. Adaptive synchronization scheme is proposed by designing appropriate controllers and parameter updating laws. Based on Lyapunov stability theory, synchronization results are obtained. The method is applied to Lorenz and hyper-chaotic Chen systems, respectively. Corresponding numerical simulations show the effectiveness of the method proposed. In existing literatures results about synchronization between chaotic and time-delayed chaotic systems are still few. So the obtained will be helpful in synchronizing chaotic and timedelayed chaotic systems.

\section{Acknowledgements}

This study is supported by the Specialized Research Fund for the Doctoral Program of Higher Education of China (No. 20093401120001), the Natural Science Foundation of An-hui Province (No. 11040606M12) and the Natural Science Foundation of Anhui Education Bureau (No. KJ2010A035), the 211 project of Anhui University(No. KJJQ1102).

\section{Authors' contributions}

RC W directed the study, helped inspection, established the models, carried out the results of this article and drafted the manuscript. $J L L$ performed the numerical simulation. All the authors read and approved the final manuscript.

\section{Competing interests}

The authors declare that they have no competing interests.

Received: 7 January 2012 Accepted: 28 March 2012 Published: 28 March 2012

References

1. Pecora, LM, Carroll, TL: Synchronization in chaotic systems. Phys Rev Lett. 64, 821-824 (1990). doi:10.1103/ PhysRevLett.64.821

2. Zhang, J, Li, CG, Zhang, HB, Yu, JB: Chaos synchronization using single variable feedback based on backstepping mathod. Chaos Solitons Fract. 21, 1183-1193 (2004). doi:10.1016/j.chaos.2003.12.079

3. Shuai, JW, Durand, DM: Phase synchronization in two coupled chaotic neurons. Phys Lett A. 264, 289-297 (1999). doi:10.1016/S0375-9601(99)00816-6

4. Ge, ZM, Chang, CM: Generalized synchronization of chaotic systems by pure error dynamics and elaborate Lyapunov function. Nonlinear Anal TMA. 71, 5301-5312 (2009). doi:10.1016/j.na.2009.04.020

5. $\mathrm{Xu}, \mathrm{YH}$, Zhou, WN, Fang, JA, Sun, W: Adaptive lag synchronization and parameters adaptive lag identification of chaotic systems. Phys Lett A. 374, 3441-3446 (2010). doi:10.1016/j.physleta.2010.06.064

6. Ghosh, D, Bhattacharya, S: Projective synchronization of new hyperchaotic system with fully unknown paramaters. Nonlinear Dyn. 61, 11-21 (2010). doi:10.1007/s11071-009-9627-4

7. Park, JH: Adaptive control for modified projective synchronization of a four-dimentional chaotic system with uncertain parameters. J Comput Appl Math. 213, 288-293 (2008). doi:10.1016/j.cam.2006.12.003

8. Park, JH: Adaptive modified projective synchronization of a unified chaotic system with an uncertain parameter. Chaos Solitons Fract. 34, 1552-1559 (2007). doi:10.1016/j.chaos.2006.04.047

9. Luo, RZ, Wei, ZM: Adaptive function projective synchronization of unified chaotic systems with uncertain paramaters. Chaos Solitons Fract. 42, 1266-1272 (2009). doi:10.1016/j.chaos.2009.03.076

10. Du, HY, Zeng, QS, Wang, CH, Ling, MX: Function projective synchronization in coupled chaotic systems. Nonlinear Anal RWA. 11, 705-712 (2010). doi:10.1016/j.nonrwa.2009.01.016

11. Park, JH: Further results on functional projective synchronization of Genesio-Tesi chaotic system. Modern Phys Lett B. 24, 1889-1895 (2009). doi:10.1142/S0217732309031302

12. Lee, TH, Park, JH: Adaptive funcrional projective lag synchronization of hyperchaotic Rössler system. Chin Phys Lett. 26, 090507 (2009). doi:10.1088/0256-307X/26/9/090507

13. Shi, XR, Zuo, LW: Adaptive added-order anti-synchronization of chaotic systems with fully unknown parameters. Appl Math Comput. 11, 1711-1717 (2009)

14. Mainieri, R, Rehacek, J: Projective synchronization in three-dimensional chaotic systems. Phys Rev Lett. 82, 3042-3045 (1999). doi:10.1103/PhysRevLett.82.3042

15. Li, GH: Modified projective synchronization of chaotic system. Chaos Solition Fract. 32, 1786-1790 (2007). doi:10.1016/j. chaos.2005.12.009

16. Chen, Y, Li, X: Function projective synchronization between two identical chaotic systems. Int J Modern Phys C. 18, 883-888 (2007). doi:10.1142/S0129183107010607

17. Du, HY, Zeng, QS, Wang, CH: Modified function projective synchronization of chaotic system. Chaos Solitions Fract. 42, 2399-2404 (2009). doi:10.1016/j.chaos.2009.03.120 
18. Sudheer, KS, Sabir, M: Adaptive modified function projective synchronization between hyper-chaotic Lorenz system and hyperchaotic Lü system with uncertain parameters. Phys Lett A. 373, 3743-3748 (2009). doi:10.1016/j. physleta.2009.08.027

19. Li, LX, Peng, HP, Yang, YX, Wang, XD: On the chaotic synchronization of 694 Lorenz systems with the time-delayed lags. Chaos Solitons Fract. 41, 783-794 (2009). doi:10.1016/j.chaos.2008.03.014

20. Feng, CF, Zhang, Y, Sun, JT, Qi, W, Wang, YH: Generalized projective synchronization in time-delayed chaotic systems. Chaos Solitons Fract. 38, 743-747 (2008). doi:10.1016/j.chaos.2007.01.037

21. Sudheer, KS, Sabir, M: Adaptive modified function projective synchronization of multiple time-delayed chaotic Rössler system. Phys Lett A. 375, 1176-1178 (2011). doi:10.1016/j.physleta.2011.01.028

22. Ghosh, D, Chowdhury, AR: Dual-anticipating, dual and dual-lag synchronization in modulated time-delayed systems. Phys Lett A. 374, 3425-3436 (2010). doi:10.1016/j.physleta.2010.06.050

23. Pourdehi, S, Karimaghaee, P, Karimipour, D: Adaptive controller design for lag-synchronization of two non-identical time-delayed chaotic systems with unknown parameters. Phys Lett A. 375, 1769-1775 (2011). doi:10.1016/j. physleta.2011.02.008

24. Grzybowski, JMV, Macau, EEN, Yoneyama, T: Isochronal synchronization of time delay and delay-coupled chaotic systems. J Phys A Math Theory. 44(17):175103 (2011). doi:10.1088/1751-8113/44/17/175103

25. Illing, L, Panda, CD, Shareshian, L: Isochronal chaos synchronization of delay-coupled optoelectronic oscillators. Phys Rev E Stat Nonlinear Soft Matter Phys. 84, 016213 (2011)

26. Ghosh, D: Projective synchronization in multiple modulated time-delayed systems with adaptive scaling factor. Nonlinear Dyn. 62, 751-759 (2010). doi:10.1007/s11071-010-9759-6

27. Feng, CF: Projective synchronization between two different time-delayed chaotic systems using active control approach. Nonlinear Dyn. 62, 453-459 (2010). doi:10.1007/s11071-010-9733-3

doi:10.1186/1687-1847-2012-37

Cite this article as: Wu and Liu: Isochronal function projective synchronization between chaotic and time-delayed

chaotic systems. Advances in Difference Equations 2012 2012:37.

\section{Submit your manuscript to a SpringerOpen ${ }^{\circ}$ journal and benefit from:}

- Convenient online submission

- Rigorous peer review

- Immediate publication on acceptance

- Open access: articles freely available online

- High visibility within the field

- Retaining the copyright to your article

Submit your next manuscript at $\gg$ springeropen.com 